\title{
Real-Time Whiteboard Capture System
}

\author{
Awab Fakih ${ }^{1}$, Jovita Serrao ${ }^{2}$ \\ ${ }_{1,2}^{2}$ (Electronics and Telecommunication, Mumbai University, India)
}

\begin{abstract}
Real-Time Whiteboard Capture System captures whiteboard lecture video in real time and extracts newly written pen strokes. Unlike other systems, it does not require special instruments such as electronic pen or whiteboard. The captured video images are classified into pen strokes on the whiteboard, foreground (person in front of the whiteboard) and Whiteboard Background Model (whiteboard without the text). The newly written pen strokes are extracted from current image frame and updated to the Whiteboard Background Model. Whiteboard Balancing is done where the colour of the whiteboard is corrected and made completely white. The captured video is processed at 8 frames per second (fps) instead of 25 fps that is output from the camera. Whiteboard balancing and reducing the processing frame rate provide a reduced bandwidth when transmitting whiteboard contents to remote meeting participants. This system can be used in conjunction with other teleconferencing tools such as voice conference and application sharing to improve online meeting experience.
\end{abstract}

Keywords: Whiteboard, real-time, luminance, pixels, archiving, foreground, bandwidth.

\section{INTRODUCTION}

Whiteboards, today, are an indispensable tool for sharing of ideas and allowing participants to focus their attention. It is an effective, economical and natural tool. Whiteboards are most commonly used in meeting scenarios such as brainstorming sessions, lectures, project planning and business meetings. The most common problem faced with whiteboard sessions is that content on the board is difficult to archive or share with members who are not present at the session. In addition, people who are present are often busy copying and making notes. As a result, they spend much less time absorbing ideas and understanding concepts than they should. Further, participants who are attending whiteboard sessions on a conference call from remote locations are not able to see whiteboard contents as well as do local participants. In order to enhance the experience, many a times, expensive video conferencing equipments are installed which can be controlled by remote participants.

The Real-Time Whiteboard Capture System aims to firstly eliminate the need of manual note-taking by participants by automatically capturing and saving the contents. Secondly transmit the whiteboard contents to remote meeting participants by using a low bandwidth [1], [2].

The paper is organized as follows. Section II discusses related works. Section III explains the system design considerations and Section IV presents the architecture of our system. The applications are mentioned in Section V. We discuss the future scope of our system in Section VI and conclude in Section VII.

\section{Related Work}

Some other devices, namely Whiteboard Capture Devices are used mainly for archiving whiteboard contents. These capture the whiteboard by a click of a button. Later, it may be printed or photocopied. But, the drawback with these devices is that they require user intervention whenever whiteboard has to be captured. The user has to make conscious decision to take snapshot of the whiteboard. Thus these devices are not very convenient in teleconferencing environment [3].

Pen Tracking Devices are another category that tracks the location of pen on the whiteboard and infers contents of whiteboard from the history of pen coordinates. The pen emits ultrasonic pulses whenever pressed against the board. Two receivers are used to detect difference in time-of-arrival of the audio pulses. The history of pen coordinates is used to construct whiteboard content in real time. These devices have the disadvantage that they either require special ultrasonic pens and erasers or the whiteboard needs to be instrumented.

Most existing systems for the purpose of capturing whiteboard content require special electronic pens or computer programmed whiteboards. They may also require user intervention whenever the whiteboard content has to be captured [3]. Our method allows the user to write freely and eliminates the need of specialized pen or whiteboard. To achieve this, an off-the-shelf high-resolution video camera (Logitech HD Pro WebCam C910) is used to capture images of the whiteboard which are processed at $8 \mathrm{~Hz}$. The input video sequence is analyzed to separate people in the foreground from the whiteboard background and extracts the pen strokes as they are written on the whiteboard. The images are also white-balanced and colour-enhanced for greater compression rate and better viewing experience than 
III. System Design

The system can be divided into three basic blocks as shown in Fig. 3.1.

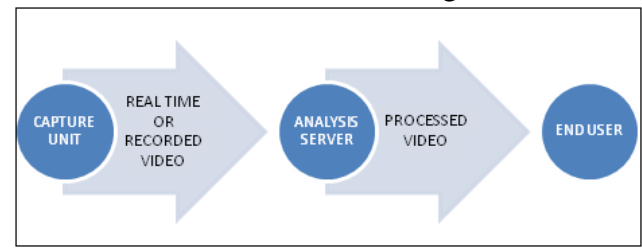

Fig. 3.1: Basic block diagram

\subsection{Capture Unit}

A capture unit is installed in the room where meetings take place. It consists of a HD web camera (Logitech HD Pro C-910 in our case), an external or built in microphone, and a PC. The web camera is interfaced to the MATLAB software on the PC where Analysis Server is located. The main function of the capture unit is to take input of whiteboard lectures in real time and send it to the analysis server.

\subsection{Analysis Server}

Analysis server is located in a central place where the entire processing is done on the captured video. It also stores the recorded video. In case of real time processing, an analysis program is executed automatically once the capture unit starts capturing the video. In case of real time, the original video as captured from the camera is not saved. In case processing is being done for recorded video, a separate video file is created which saves the processed frames at the location from where recorded video is browsed. Hence all the processed videos are archived; both those that are captured in real time and those that are processed using previously recorded whiteboard lectures.

\subsection{End User}

The video being processed in real-time can be viewed by the users who are present at the venue of the lecture and even those who maybe attending the lecture at remote location over a network. If the user wishes to process any previously recorded video then a GUI is available which will help the user to browse the recorded video and then process it [1], [2].

\section{Technical Details}

The design goals of the Real time Whiteboard Capture System are to work with any existing whiteboard. Capture the whiteboard content automatically and reliably. Use the whiteboard content as a visual index to efficiently browse the recorded meeting.

The input to RTWCS is a sequence of video images as shown in Fig. 4.1. We need to analyze the image sequence in order to separate the whiteboard background from the person in the foreground and to extract the new pen strokes as they appear on the whiteboard. However, our system has a set of unique technical challenges. The whiteboard background colour cannot be pre-calibrated (e.g., take a picture of a blank whiteboard) because each indoor room has several light settings that may vary from session to session and outdoor room lighting condition is influenced by the weather and the direction of the sun. Frequently, people move between the camera and the whiteboard, and these foreground objects occlude some portion of the whiteboard and cast shadow on it. Within a sequence, there may be no

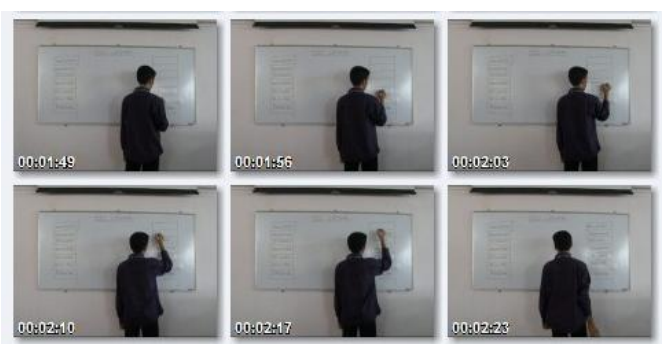

Fig. 4.1: Selected frames from an input image sequence. The duration of the sequence is 34 second single frame that is completely unoccluded. A few sample image frames are shown in Fig. 4.1. We need to deal with these problems in order to extract the new pen strokes.

The RTWCS does not require people to move out of the camera's field of view during capture as long as they do not block the same portion of the whiteboard during the entire meeting. The system does not require any special installation or calibration. The video camera can be placed anywhere that has a steady and clear view of the whiteboard. Although the camera can be placed anywhere, the intended capture area should occupy 
as much video frame as possible in order to maximize the available image resolution. For better image quality, it is also better to place the camera right in front of the whiteboard. The RTWCS system has been developed for both real time as well as recorded videos [4], [5].

\subsection{Interfacing The Web Camera}

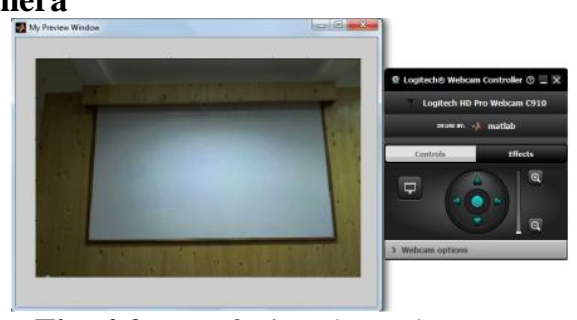

Fig. 4.2: Interfacing the web camera

The input device is a web camera having resolution 1920 pixels by 1080 pixels. The camera is connected to any PC with a USB 2.0 port, as shown in Fig. 4.2. The input taken from the camera is directly processed and the output video is saved on the desktop of the PC.

\subsection{Whiteboard Extraction}

Three corners of the whiteboard are manually selected. A rectangle is created using the obtained three vertices and then the whiteboard is cropped in accordance to this rectangle as shown in Fig. 4.3. This method for extracting whiteboard gives best result and reduces the processing load as the portion of whiteboard is only processed and not the background.

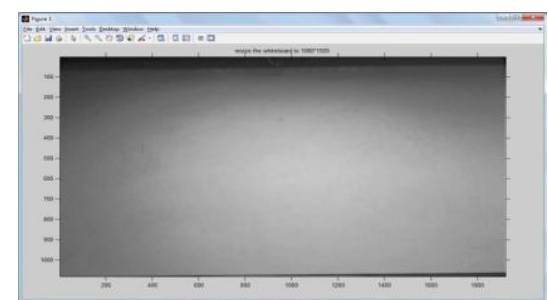

Fig. 4.3: Extracted whiteboard

\subsection{Creation Of Whiteboard Background Model}

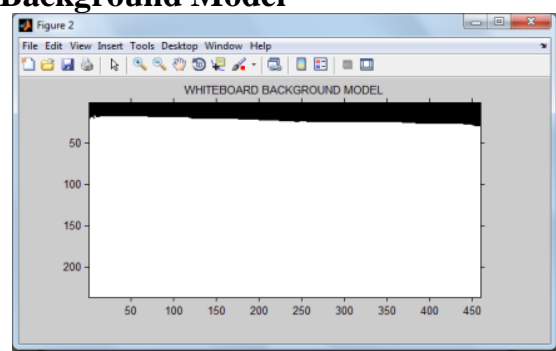

Fig. 4.4: Whiteboard background model

The whiteboard background model as in Fig. 4.4 is the colour of the whiteboard without anything written on it [6]. The luminance of the whiteboard pixels is higher than the pen stroke pixels. The whiteboard colour is the colour in the frame with the highest luminance. All the pixels having luminance above a set threshold are considered to be whiteboard pixels. The obtained pixels are then filtered to refine the existing whiteboard background model.

\subsection{Updating The Whiteboard}

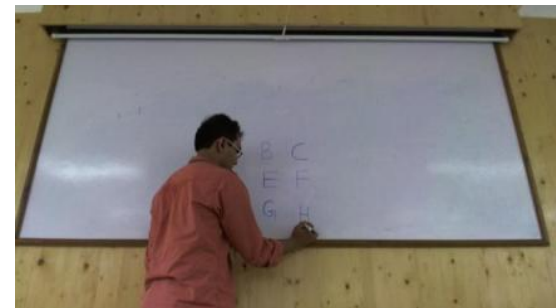

Fig. 4.5: Screen shot of the original video 


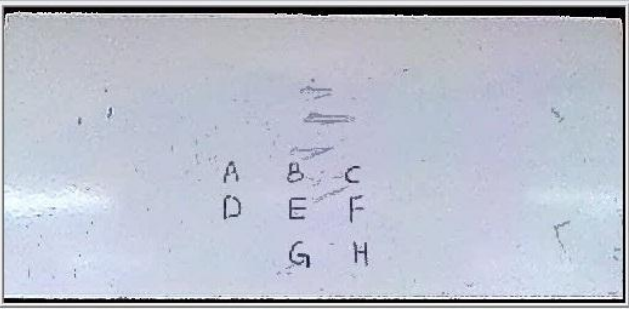

Fig. 4.6: Screen shot of the processed video

The first frame is considered to be the reference frame. The whiteboard background model is created from this reference frame. The current frame is then compared to the reference frame. The frames are converted to binary format and then processed. The red, blue and green frames are processed separately. The pen strokes are obtained from the current frame and updated on the reference frame. The obtained frame is then filtered to refine the pixels [7], [8]. A screen shot of the original frame is shown in Fig. 4.5. Fig. 4.6 is the screen shot of the processed video after updating the whiteboard. It can be seen that even the portion of the board covered by the person in front of it is visible on the processed board.

\subsection{Archiving Of Processed Video}

If the video input is obtained by interfacing the web camera to the software, the processed video is saved on the desktop of the PC in the audio video interleaved (AVI) format. For recorded videos, the processed video is saved in the same folder as the original video [9], [10].

\section{$5.1 \quad$ Online Videoconferencing}

\section{Applications}

The main application of our system is in online videoconferencing to facilitate remote participants to easily read the text written on whiteboard or on presentation slides. They may also save the whiteboard as an image at any point which eliminates the need for them to take handwritten notes of the videoconference. They can hence focus on the main subject matter of the conference.

\subsection{Business Meetings}

In a business meeting environment, this system can be installed. By this the attendants will not require to take notes of the whiteboard writings. They may be given a summary of the same at the end of the meeting by saving and printing pictures at points in the meeting whenever the entire whiteboard is erase. Also if the number of attendants is large, we may display the whiteboard contents far away from the location of the speaker after processing it through the system.

\subsection{Distance Education}

Distance education is an initiative by virtue of which students can undertake courses of universities at remote locations. The students are supplied by the required text to prepare for exams. This system will be an enhancement feature to the concept of distance education where students will be capable of remotely attending lectures in addition to the text provided to them for preparation of exam. This will help in clear understanding of concepts which they may not get from the text alone.

\subsection{Archiving Of Meetings}

Meetings are usually archived in the form of complete videos. This requires more memory space and is also inconvenient to review. By storing the meetings in the form of whiteboard content at certain time intervals will reduce the amount of memory needed to save the meeting and also help easy revision.

\section{Future Scope}

The system may be further enhanced by integration of additional interactive audio visual features. For example, giving the remote participant a facility to voice his queries to be transmitted to the meeting location or write them in 'notepad' or 'paint' and transmit it to be projected on the whiteboard. This will be a step towards a more dynamic and mutual meeting session and clarify points that maybe in doubt.

\section{Conclusion}

The system presented in this report improves the interaction experience of any business meeting, lecture, brainstorming session or project planning for those present at the meeting location as well as remote participants. Since participants are no longer concerned about copying the whiteboard content, which is being processed by the system and archived, it results in a more efficient use of time for exchange of ideas. This 
provides for a better understanding and positively influences their productivity. For remote participants, in addition to whiteboard content archiving, it improves the visual experience of the meeting as the whiteboard is seen without being occluded by foreground objects (such as the presenter).

Since only the contents of the whiteboard are extracted and transmitted, it requires lower bandwidth compared to expensive video-conferencing solutions. It uses a low-cost video camera to input whiteboard video. The system also eliminates the need of specialized markers and whiteboards and makes the interaction more natural. These features make it a promising solution for scenarios where whiteboard is used.

\section{References}

[1] L He and Z Zhang, Real-time whiteboard capture and processing using a video camera for remote collaboration, IEEE Trans Multimedia, vol 9, pp 198-206, January 2007

[2] L He and Z Zhang, Real-time whiteboard capture and processing using a video camera for teleconferencing, in Proc ICASSP, vol 2, pp 1113-1116, 2005

[3] L He, Z Liu, and Z Zhang, Why take notes? Use the whiteboard capture system, Proc ICASSP, vol 5, pp 776-779, 2003

[4] H S Malvar, L He, and R Cutler, High-quality linear interpolation for demosaicing of bayer-patterned colour images, Proc ICASSP, vol 3, pp 485-488, 2004

[5] E. Saund, Image mosaicing and a diagrammatic user interface for an office whiteboard scanner, Tech Rep, Xerox Palo Alto Research Center, 1999

[6] Peter Meer, Charles V. Stewart and David E. Tyler, Robust computer vision: an interdisciplinary challenge, in Computer vision and image understanding, vol 78, pp 1-7, 2000

[7] R.C.Gonzalez, Digital Image Processing, Prentice Hall Publication, Third Ed., 2008

[8] Ramesh Jain, Rangachar Kasturi and Brian G. Schunk, Machine Vision, McGraw-Hill Science, 1995

[9] Rousseeuw, P. and Leroy, A Robust Regression and Outlier Detection, John Wiley \& Sons, 1987

[10] S X Ju, M J Black, S Minnerman, and D Kimber, Summarization of video-taped presentations: Automatic analysis of motion and gesture, IEEE Trans Circuits Syst Video Tech, vol 8, no 5, pp 686-696, September 1998 